INPLASY

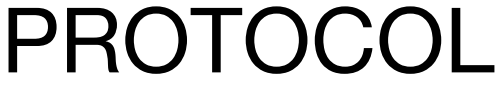

To cite: Wang et al.

Acupuncture for atraumatic shoulder conditions: protocol for a systematic review and meta-analysis. Inplasy protocol 202140096. doi:

10.37766/inplasy2021.4.0096

Received: 18 April 2021

Published: 18 April 2021

Corresponding author:

Yi Wang

yiwang1984@hotmail.com

Author Affiliation:

Sichuan Provincial

Orthopedics Hospital

Support: No funding was secured.

Review Stage at time of this submission: The review has not yet started.

Conflicts of interest:

None declared.

\section{Acupuncture for atraumatic shoulder conditions: protocol for a systematic review and meta-analysis}

Review question / Objective: To assess the effectiveness of acupuncture in the treatment of atraumatic shoulder conditions.

Condition being studied: Atraumatic shoulderconditions (including rotator cuff tear from minor trauma, rotator cuff tendinopathy, subacromial impingement, osteoarthritis of the glenohumeral and/or acromioclavicular joint, adhesive capsulitis, frozen shoulder, calcific tendinitis, tendinopathy and tenosynovitis of the long head of the biceps brachii, shoulder bursitis, and shoulder pain where the cause has not been specifie) as diagnosed by clinical examination and/or diagnostic imaging (e.g. ultrasonography (US), magnetic resonance imaging (MRI) or magnetic resonance arthrography (MRA)).

INPLASY registration number: This protocol was registered with the International Platform of Registered Systematic Review and Meta-Analysis Protocols (INPLASY) on 01 April 2021 and was last updated on 01 April 2021 (registration number INPLASY202140001).

\section{INTRODUCTION}

Review question / Objective: To assess the effectiveness of acupuncture in the treatment of atraumatic shoulder conditions

Condition being studied: Atraumatic shoulderconditions (including rotator cuff tear from minor trauma, rotator cuff tendinopathy, subacromial impingement, osteoarthritis of the glenohumeral and/or acromioclavicular joint, adhesive capsulitis, frozen shoulder, calcific tendinitis, tendinopathy and tenosynovitis of the long head of the biceps brachii, shoulder bursitis, and shoulder pain where the cause 
has not been specifie) as diagnosed by clinical examination and/or diagnostic imaging (e.g. ultrasonography (US), magnetic resonance imaging (MRI) or magnetic resonance arthrography (MRA)).

\section{METHODS}

Participant or population: Patients with atraumatic shoulder conditions treated non-surgically.

Intervention: Any type of acupuncture will be included, such as acupuncture, electroacupuncture, elongated needle acupuncture, internal heat-type acupuncture, intradermal Needleembedding therapy, warm acupuncture, fire needle acupuncture, auricular acupuncture, abdominal acupuncture, acupoint injection and floating needle therapy.

Comparator: Control interventions may include no treatment, sham acupuncture (acupuncture the point which is not an acupuncture point), physical therapy, nonsteroidal anti-inflammatory drugs, analgesic agents and local injection of steroid and/or local anesthetics.

Study designs to be included: Only randomized clinical controlled trials will be included.

Eligibility criteria: Adults ( $>18$ years of age) with atraumatic shoulder conditions. Excluded will be studies among populations with 'red flag' diagnoses (e.g. rheumatoid arthritis, tuberculosiscancer, infection, cancer); studies among populations with rotator cuff tear from major trauma (e.g. sports injury, car accident, fall from a height that exceeds one's own height) or with other major trauma-related conditions; studies on populations with glenoid labrum pathologies, previous surgery at the affected shoulder, neck disorders, multisite musculoskeletal pain, relevant systemic diseases and disorders, or neurologic disorders.
Information sources: The following databases will be searched from January 2000 till May 1 2021: PubMed, EMBASE,Cochrane Central Register of Controlled Trials, Web of Science, Ovid MEDLINE, Chinese Biomedical Literature Database,Chinese National Knowledge Infrastructure, Wanfang Database, and Chongqing VIP database. No restriction on language. Information from articles not in the English or Chinese language will be translated using Google's translation tools to acquire relevant information where available.

Main outcome(s): The main (primary) outcome is shoulder pain intensity, measured by visual analogue scale (VAS) score or numeric rating scale (NRS). The results will be expressed as percentages to describe the distributions and $95 \% \mathrm{Cl}$ to measure the reliability. The $x^{2}$ test and the $I^{2}$ were applied to evaluate heterogeneity of studies. And $95 \% \mathrm{Cl}$ will be used to measure the reliability. Means and SDs will be calculated to describe continuous variables.

Additional outcome(s): Additional outcomes are shoulder function, measured by validated scales, such as should range of motion (ROM), the Simple Shoulder Test (SST) score, Constant-Murley score(CMS), Shoulder Pain and Disability Index (SPADI) total scale, Dutch Shoulder Disability Questionnaire (SDQ-NL), University of California-Los Angeles (UCLA) Shoulder rating scale; health-related quality of life measured on validated scales such as Short-Form 36 (SF-36) Health Survey or EuroQoL EQ-5D; and occurrence of adverse events. The results will be expressed as percentages to describe the distributions and $95 \% \mathrm{Cl}$ to measure the reliability. The $X^{2}$ test and the $I^{2}$ were applied to evaluate heterogeneity of studies. And $95 \% \mathrm{Cl}$ will be used to measure the reliability. Means and SDs will be calculated to describe continuous variables.

Data management: Two authors will independently screen for titles and abstracts. After removing duplicates, they 
will then use the full-text articles to determine which papers will finally be included in the review. If the 2 review authors disagree about whether to include a paper, a third reviewer will decide on whether the paper will be included. Two authors will independently extract relevant data from each of the eligible studies using a standardized data extraction form. The following information will be extracted: first author's name, date of publication, country, language, age, gender, sample size duration of disease, severity of symptoms at baseline, followup, acupuncture and control intervention (e.g., the specific acupuncture methods, number of sessions, duration, acupuncture points), outcome measures, results of intervention, duration of follow-up assessments, and reported adverse effects. We will contact the corresponding author to retrieve additional information if necessary.

Quality assessment / Risk of bias analysis: The quality of the included trials will be evaluated by 2 authors using the Cochrane Collaboration's tool. Seven domains (randomly generated sequence number, allocation concealment, blinding of participants, blinding of outcome assessment, incomplete outcome data, selective reporting, and other bias when required) will be assessed. For each aspect, the trial will be rated as high, low risk, or unclear of bias. A trial that is rated as high risk of bias in 1 or more aspects will be graded as'high risk', while a low risk of bias in all aspects will be graded as'low risk'. If there is a low or unclear risk of bias for all main aspects, the trial will be rated as 'unclear risk'. The contact person or corresponding author will be contacted if basic information is missing for the risk of bias assessment. The rating results will be cross-checked and discrepancies resolved through discussions and the arbitration of a third author.

Strategy of data synthesis: A meta-analysis will be performed using RevMan software (Review Manager Version $\mathbf{5 . 3}$ for Windows, The Nordic Cochrane Centre, Copenhagen). A random effects model will be used to calculate the pooled effect estimates, because substantial clinical heterogeneity is expected among the studies included in this review. If considerable heterogeneity $(12>75 \%)$ is observed, we will not meta-analyze the trials and will qualitatively synthesize the data.

Subgroup analysis: If an adequate number of studies are available, subgroup analysis will be performed to find possible sources of the heterogeneity. Classifications are as follows: (1). gender of the study population; (2). age range of the study population: less than 40 years old, $40-60$ years old, more than 60 years old; (3). the specific acupuncture methods (i.e. acupuncture, electroacupuncture, elongated needle acupuncture, internal heat-type acupuncture, intradermal Needleembedding therapy, warm acupuncture, fire needle acupuncture, auricular acupuncture, abdominal acupuncture, acupoint injection and floating needle therapy).

Sensitivity analysis: Sensitivity analysis will be performed by sequentially excluding each study.

Country(ies) involved: China.

Keywords: acupuncture; shoulder; systematic review.

Contributions of each author:

Author 1 - Yi Wang.

Email: yiwang1984@hotmail.com

Author 2 - Yan Xu.

Email: 905152285@qq.com

Author 3 - Yu Peng.

Email: 1152317594@qq.com

Author 4 - Guogang Dai.

Email: guogang_dai@hotmail.com

Author 5 - Shichuan Liao.

Email: 648663372@qq.com

Author 6 - Jiao Xia.

Email: summer6470@163.com

Author 7 - Guogang Tian.

Email: tianguogang123@hotmail.com 